\title{
The Relationship of the Manufacturing Growth with the Financial Industry and Real Estate Industry
}

\author{
Yueyun (Bill) Chen* \\ University of the West, Los Angeles, USA \\ Xingong Li* \\ Henan University, China \\ Chengyi Pu \\ Southwest Minzu University, China \\ Lianlian Lin \\ California State Polytechnic University Pomona, USA
}

\begin{abstract}
This paper focuses on the relationship of the manufacturing industry with the financial and real estate industry.The purpose is to examine whether the fast development of the financial or real estate sector negatively affects the growth of the manufacturing industry as some claimed. Based on the data of China, USA, Germany, and Japan and using the regression analysis, the paper identified factors or variables significantly affecting a country are manufacturing growth. However, it did not find out any such direct significant effect of the financial or real estate sector on the manufacturing industry. On the other hand, the productivity, export, and investment of the manufacturing industry are more important generally to the growth of manufacturing. The paper then further discusses and suggests policy implications on what a country should do to maintain sustainable manufacturing growth.
\end{abstract}

\section{JEL Code: E00, O11, O57, R30, L00}

Keywords: Manufacturing industry, Financial industry, Real estate industry, Productivity, Economic growth

The shrinking of the manufacturing industry and the decrease of jobs in the sector and whole economy have been a serious problem in developed countries as well as some developing countries like China (Chen et al. 2017). What caused such a significant decrease of the manufacturing industry? People often blame fast developments of the financial industry and real estate sector. They argue that the investment goes to these fast-growing sectors due to the high rate of return; therefore, little money is left to be invested in the manufacturing industry. Also, the salary and benefits are better in these fast-growing sectors. As a result of these, less labor will be available in manufacturing.

This paper uses the data from China, USA, Germany, and Japan to examine whether any significant statistical relationship of developments between the manufacturing and financial or real estate sector. Then, based on the regression analysis and its outcomes, the paper makes suggestions to these four countries on what they should do in order to maintain sustainable and stable developments of the manufacturing industry.

Not many previous researches have directly addressed the relationship of the manufacturing industry with the financial and real estate industry; especially there were not much regression analyses on the subject. Besides two variablesfinancial and real estate growth, the paper also includes other variables-the productivity growth in manufacturing, investment and export growth in the industry, the R \& D spending growth of the country and Foreign Direct Investment (FDI) inflow of the country, therefore, the econometric model used in this study is more comprehensive than other previous studies.

The rest of the paper is organized as follows: next section reviews the literature; Section II explains the data used, variables selected and provides the relevant summary statistics and correlations; Section III gives the econometric model, hypothesis, and regression outcomes; Section IV discusses the policy implications based on significant variables from the regressions and particularly discusses how countries like China can better sustain its manufacturing growth; Section V concludes the paper and reviews possible future research.

\section{Review of literature}

According to the economic production theory, the economic output is based on its inputs of labor, capital, and other factors such as technological progress and innovations. 
Therefore, the growth of manufacturing relies on the changing of these inputs. One may also view an industry's growth from the demand and supply side. The manufacturing sector shrinks because of less demand for its products and at least less demand for the domestic-made products. As a result, a country's manufacturing sector falls. From the view point of the supply-side, when less companies are willing to produce these manufacturing products, the whole sector drops. The reasons for such unwillingness can be the production-cost or profit margin related or due to the opportunity costs.

International trade theory explains trades and shifting of manufacturing companies among countries based on absolute and/or comparative advantages of the costs. Manufacturing was outsourced from developed countries like the US to developing countries like China because of the cost-saving in doing so. When production cost increases significantly in China, these manufacturers will shift to other places like Southeastern countries. As a result, China's manufacturing suffers. The competitive advantage theory shows that a manufacturer's growth depends on whether it can sustain its competitive advantages against its competitors. These advantages can be cost-related or others such as quality or technology.

There have had studies on the relationship between the manufacturing development and economic growth. Baily and Bosworth, B. P. (2014) had comprehensive review of US manufacturing developments in the past and future and its effects on the economy. Dehejia and Panagariya (2014) studied the link of manufacturing and service industries developments in India. Kerwin et al. (2016) explained how house market bubbles affected manufacturing employments. Chen (2015) demonstrated how its manufacturing developments helped China's exports and economic growth and how crucial the sustainable and stable manufacturing growth will be to China's future economic development.

There were studies on the relationship between the development of financial industry and economic growth. Cezar (2014) studied the effects of finance on international trade. Goldsmith (1969), McKinnon (1973) and Shaw (1973) showed that the development of financial institutions partly explained different economic growth rates in different countries. King and Levin (1992, 1993a, 1993b) explained the effects of financial institutions on entrepreneurship and innovations and therefore on the productivity and the economic growth. But Robinson (1952), Lucas (1988), and Stern (1989) doubted the importance of financial factors in growth. Solow $(1956,1957)$ viewed the changes of investment having only minor effects on growth.

Although there were not many studies on the direct relationship between the manufacturing and real estate, there were some studies on the relationship between the manufacturing growth and financial industry. Anwar and Sun (2013) focused on how Foreign Direct Investments (FDIs) affected China's manufacturing industry. Meusserand Kugler(1998) found that there were not many co-integrations between the manufacturing outputs and financial sector GDP among OECD countries; however, they found some links between the manufacturing Total Factor Productivity (TFP) and financial development although such links are complex in different countries.

This paper studies the relationship of the manufacturing growth with both financial and real estate industry. The contributions of the paper are that it includes the real estate sector and also compares the effects in developed countriesUS, Germany, and Japan and developing country-China. Also, this paper uses regression models to study the relationship, different from many previous approaches which used co-integration methods. The advantage of using the regression models is that it can control effects of other relevant variables. In addition, it can show which factors affect the manufacturing growth more significantly. The most important contribution of this paper is its policy applications. Based on this study, main factors affecting a country's manufacturing growth are different, given its development stage and unique situations. Then each country can focus on its main problems to improve its manufacturing growth. In addition, a developing country like China can learn from other developed countries and adjust its policy and actions to maintain its manufacturing strength when its economy advances toward a high income economy.

\section{Variables, data, summary statistics and correlations}

Based on the economic theory and previous other relevant studies, this paper uses the following variables: $\mathrm{GDP}_{\mathrm{m}}$ is the country's annual manufacturing growth rate; INVEST $\mathrm{M}_{\mathrm{M}}$, the annual investment growth rate in manufacturing; PRODUCT $_{\mathrm{M}}$, the annual productivity growth of the manufacturing, where the productivity is the GDP in manufacturing divided by the \# of employees in the sector; EXPORT $\mathrm{E}_{\mathrm{M}}$, the annual manufacturing export growth rate; $\mathrm{GDP}_{\mathrm{F}}$, the annual GDP growth rate of the financial sector; $\mathrm{GDP}_{\mathrm{R}}$ the annual GDP growth rate of the real estate sector; $\mathrm{R} \& \mathrm{D}$, the annual growth rate of a country's R\&D spending; and FDI, the annual growth rate of the Foreign Direct Investment inward(inflow). In addition, GDP is the annual GDP growth rate of the relevant country.

The annual growth rates are calculated and used for all variables. The growth rates should be more stable and better than absolute values and particularly they will be less influenced by the exchange rate changes in specific years for specific countries. 
China, USA, Germany, and Japan are selected since they are the top four largest manufacturing countries and also the largest economies in the world. Except investment data, all data of the relevant variables of these four countries over years are collected from the World Bank unless otherwise mentioned (please see the Endnotes).

\subsection{Summary statistics of data}

Table 1 is the summary statistics of China's data; Table 2 is for the USA; Table 3 for Germany and Table 4 for Japan.

Table 1. Summary of China Data

\begin{tabular}{|c|c|c|c|c|c|c|c|c|c|}
\hline Year & $\mathbf{G D P}_{\mathbf{M}}$ & GDP & INVEST $_{M}$ & PRODUCT $_{M}$ & $\mathbf{G D P}_{\mathbf{F}}$ & $\mathbf{G D P}_{\mathbf{R}}$ & EXPORT $_{M}$ & R\&D & FDI \\
\hline 1991 & 0.1224 & 0.1136 & & 0.0455 & 0.1312 & 0.1533 & 0.2282 & 0.1191 & 1.5552 \\
\hline 1992 & 0.0815 & 0.0417 & -0.3341 & 0.0958 & 0.1484 & 0.4421 & 0.1060 & -.0054 & 1.4664 \\
\hline 1993 & 0.2567 & 0.2689 & 2.1509 & 0.0374 & 0.0113 & 0.2527 & 0.3467 & 0.1493 & 0.2279 \\
\hline 1994 & 0.3028 & 0.3016 & 0.1795 & 0.2283 & 0.2633 & 0.3840 & 0.2567 & 0.1751 & 0.0610 \\
\hline 1995 & 0.1710 & 0.1759 & -0.4355 & 0.2743 & 0.2747 & 0.2329 & 0.0180 & 0.1651 & 0.1208 \\
\hline 1996 & 0.1019 & 0.1133 & -0.4040 & 0.1314 & 0.1730 & 0.1120 & 0.2245 & 0.2625 & 0.1010 \\
\hline 1997 & 0.0269 & 0.0701 & -0.7364 & 0.0790 & 0.1609 & 0.1159 & 0.0278 & 0.0838 & -0.0110 \\
\hline 1998 & 0.0543 & 0.0631 & 1.0206 & 0.0236 & 0.1324 & 0.1758 & 0.0730 & 0.2320 & -0.1142 \\
\hline 1999 & 0.1248 & 0.1073 & 1.2996 & 0.0658 & 0.1071 & 0.0720 & 0.2775 & 0.3193 & 0.0862 \\
\hline 2000 & 0.0888 & 0.1057 & -0.2887 & 0.1388 & 0.1421 & 0.1269 & 0.0725 & 0.1641 & 0.1178 \\
\hline 2001 & 0.0897 & 0.0979 & 0.0107 & 0.0878 & 0.1456 & 0.1364 & 0.2407 & 0.2351 & 0.1280 \\
\hline 2002 & 0.1802 & 0.1290 & 1.0085 & 0.1281 & 0.1252 & 0.1339 & 0.3569 & 0.1957 & 0.0910 \\
\hline 2003 & 0.1599 & 0.1777 & -0.1128 & 0.1621 & 0.1231 & 0.1545 & 0.3661 & 0.2772 & 0.1764 \\
\hline 2004 & 0.1734 & 0.1691 & 0.0846 & 0.1056 & 0.1540 & 0.1622 & 0.2912 & 0.2585 & 0.5284 \\
\hline 2005 & 0.2174 & 0.2039 & 0.2534 & 0.1036 & 0.1734 & 0.1871 & 0.2786 & 0.2597 & 0.1919 \\
\hline 2006 & 0.2873 & 0.2907 & 0.3217 & 0.1447 & 0.2179 & 0.2177 & 0.2690 & 0.2949 & 0.2592 \\
\hline 2007 & 0.2835 & 0.2945 & -0.0132 & 0.2049 & 0.3228 & 0.3316 & 0.1712 & 0.3621 & 0.0978 \\
\hline 2008 & 0.0924 & 0.1113 & -0.6742 & 0.2606 & 0.2933 & 0.0673 & -0.1548 & 0.2785 & -0.2360 \\
\hline 2009 & 0.1938 & 0.1939 & 1.0984 & 0.0650 & 0.1506 & 0.2869 & 0.3127 & 0.2282 & 0.8595 \\
\hline 2010 & 0.2583 & 0.2413 & 0.3329 & 0.1521 & 0.1870 & 0.2427 & 0.2000 & 0.2888 & 0.1492 \\
\hline 2011 & 0.1110 & 0.1305 & -0.5704 & 0.2191 & 0.2438 & 0.1951 & 0.0865 & 0.2135 & -0.1387 \\
\hline 2012 & 0.0912 & 0.1223 & -0.1785 & 0.0777 & 0.1597 & 0.1094 & 0.0792 & 0.1720 & 0.2061 \\
\hline 2013 & 0.0848 & 0.0911 & -0.0699 & 0.0945 & 0.1567 & 0.1517 & 0.0600 & 0.1081 & -0.0785 \\
\hline 2014 & 0.0208 & 0.0555 & -0.7549 & 0.0881 & 0.1738 & 0.0559 & -0.0260 & 0.0795 & -0.0955 \\
\hline 2015 & & 0.0122 & -1.0000 & 0.0390 & 0.0849 & 0.0870 & -0.0829 & & \\
\hline Average & 0.1501 & 0.1546 & 0.1386 & 0.1290 & 0.1757 & 0.1890 & 0.1710 & 0.2086 & 0.1824 \\
\hline STD & 0.0853 & 0.0801 & 0.7369 & 0.0687 & 0.0685 & 0.0997 & 0.1393 & 0.0872 & 0.3594 \\
\hline
\end{tabular}


Table 2. Summary of US Data

\begin{tabular}{|c|c|c|c|c|c|c|c|c|c|}
\hline Year & $\mathbf{G D P}_{\mathbf{M}}$ & GDP & INVEST $_{M}$ & PRODUCT $_{M}$ & $\mathbf{G D P}_{\mathbf{F}}$ & $\mathbf{G D P}_{\mathbf{R}}$ & EXPORT $_{M}$ & R\&D & FDI \\
\hline 1998 & 0.0329 & 0.0558 & 0.0782 & 0.0245 & 0.1068 & 0.0343 & 0.0080 & 0.0669 & 0.6955 \\
\hline 1999 & 0.0409 & 0.0629 & -0.0545 & 0.0552 & 0.0630 & 0.0779 & 0.0309 & 0.0820 & 0.6167 \\
\hline 2000 & 0.0409 & 0.0646 & 0.0954 & 0.0444 & 0.1058 & 0.0560 & 0.1183 & 0.0976 & 0.2094 \\
\hline 2001 & -.0500 & 0.0328 & -0.1039 & -0.0024 & 0.0900 & 0.0747 & -0.0787 & 0.0398 & -0.5102 \\
\hline 2002 & -.0043 & 0.0335 & -0.1513 & 0.0730 & 0.0204 & 0.0619 & -0.0525 & -.0012 & -0.3616 \\
\hline 2003 & 0.0394 & 0.0486 & -0.0844 & 0.0931 & 0.0363 & 0.0464 & 0.0324 & 0.0499 & 0.0172 \\
\hline 2004 & 0.0579 & 0.0664 & 0.0494 & 0.0723 & 0.0258 & 0.0549 & 0.1241 & 0.0401 & 0.8669 \\
\hline 2005 & 0.0523 & 0.0667 & 0.0531 & 0.0589 & 0.1149 & 0.0818 & 0.0984 & 0.0736 & -0.3346 \\
\hline 2006 & 0.0591 & 0.0582 & 0.1562 & 0.0643 & 0.0579 & 0.0299 & 0.1281 & 0.0768 & 1.1275 \\
\hline 2007 & 0.0281 & 0.0449 & 0.0310 & 0.0487 & -0.0086 & 0.0791 & 0.0969 & 0.0764 & 0.1556 \\
\hline 2008 & -.0216 & 0.0166 & 0.0731 & 0.0130 & -0.1305 & 0.0262 & 0.0702 & 0.0708 & -0.0216 \\
\hline 2009 & -.0531 & -0.0204 & -0.2627 & 0.0712 & 0.0704 & 0.0123 & -0.2601 & -.0020 & -0.5378 \\
\hline 2010 & 0.0607 & 0.0378 & 0.0243 & 0.0900 & 0.0382 & 0.0246 & 0.1992 & 0.0091 & 0.6864 \\
\hline 2011 & 0.0427 & 0.0370 & 0.2114 & 0.0250 & 0.0314 & 0.0362 & 0.1135 & 0.0480 & -0.0075 \\
\hline 2012 & 0.0391 & 0.0411 & 0.0573 & 0.0217 & 0.1022 & 0.0319 & 0.0410 & 0.0170 & -0.0274 \\
\hline 2013 & 0.0252 & 0.0332 & 0.0749 & 0.0174 & -0.0071 & 0.0335 & 0.0040 & 0.0470 & 0.1509 \\
\hline 2014 & 0.0320 & 0.0420 & 0.0508 & 0.0181 & 0.1014 & 0.0397 & 0.0202 & 0.0475 & -0.1752 \\
\hline 2015 & 0.0348 & 0.0418 & 0.0375 & 0.0222 & 0.0325 & 0.0509 & -0.0396 & 0.0540 & 1.1298 \\
\hline 2016 & & 0.0278 & 0.0166 & & & & -0.0465 & & -0.0528 \\
\hline Average & 0.0254 & 0.0424 & 0.0187 & 0.0450 & 0.0473 & 0.0473 & 0.0363 & 0.0496 & 0.2045 \\
\hline STD & 0.0347 & 0.0210 & 0.1127 & 0.0286 & 0.0593 & 0.0210 & 0.1032 & 0.0290 & 0.5312 \\
\hline
\end{tabular}

Table 3. Summary of Germany Data

\begin{tabular}{|c|c|c|c|c|c|c|c|c|c|}
\hline Year & $\mathbf{G D P}_{\mathbf{M}}$ & GDP & INVEST $_{M}$ & PRODUCT $_{M}$ & $\mathbf{G D P}_{\mathbf{F}}$ & $\mathbf{G D P}_{\mathbf{R}}$ & EXPORT $_{M}$ & $\mathbf{R} \& \mathbf{D}$ & FDI \\
\hline 1992 & 0.0759 & 0.1403 & 0.0241 & 0.1345 & 0.0707 & 0.2378 & 0.0691 & 0.0862 & -1.4459 \\
\hline 1993 & 0.1127 & -0.0257 & -0.0221 & -0.0572 & 0.0690 & 0.0581 & -0.1634 & -0.0569 & -1.1896 \\
\hline 1994 & 0.0411 & 0.0664 & -0.0501 & 0.1038 & 0.0151 & 0.1202 & 0.1441 & 0.0261 & 17.1651 \\
\hline 1995 & 0.1585 & 0.1748 & 1.8004 & 0.2238 & 0.1256 & 0.2290 & 0.2528 & 0.1778 & 0.6440 \\
\hline 1996 & 0.0572 & -0.0339 & -0.6732 & -0.0164 & 0.0108 & -0.0093 & -0.0254 & -0.0310 & -0.4636 \\
\hline 1997 & 0.1066 & -0.1138 & -0.0538 & -0.0938 & -0.1004 & -0.1149 & -0.0127 & -0.0966 & 0.9904 \\
\hline 1998 & 0.0211 & 0.0111 & 0.0835 & 0.0015 & -0.0442 & -0.0030 & 0.0780 & 0.0266 & 0.8471 \\
\hline 1999 & 0.0363 & -0.0193 & 0.0342 & -0.0255 & 0.1203 & -0.0485 & -0.0070 & 0.0346 & 1.3653 \\
\hline 2000 & 0.0879 & -0.1136 & -0.7480 & -0.0938 & -0.2791 & -0.0956 & -0.0162 & -0.0917 & 3.4357 \\
\hline 2001 & 0.0104 & 0.0004 & 3.4578 & -0.0080 & 0.0103 & 0.0163 & 0.0726 & -0.0022 & -0.7704 \\
\hline 2002 & 0.0384 & 0.0659 & -0.0184 & 0.0533 & 0.1361 & 0.0869 & 0.0838 & 0.0792 & -0.1003 \\
\hline 2003 & 0.2103 & 0.2052 & -0.0201 & 0.2421 & 0.2557 & 0.1972 & 0.1767 & 0.2257 & 0.2758 \\
\hline 2004 & 0.1373 & 0.1251 & -0.0690 & 0.1434 & 0.2371 & 0.1096 & 0.2043 & 0.1087 & -1.3129 \\
\hline
\end{tabular}




\begin{tabular}{|r|r|r|r|r|r|r|r|r|r|}
$\mathbf{2 0 0 5}$ & 0.0167 & 0.0150 & -0.0014 & 0.0453 & -0.0128 & 0.0244 & 0.0987 & 0.0157 & -3.9277 \\
\hline $\mathbf{2 0 0 6}$ & 0.0810 & 0.0493 & -0.0321 & 0.0639 & 0.0074 & 0.0495 & 0.1268 & 0.0638 & 0.4609 \\
\hline $\mathbf{2 0 0 7}$ & 0.1548 & 0.1457 & 0.0204 & 0.1210 & 0.0295 & 0.1652 & 0.1590 & 0.1412 & -0.4185 \\
\hline $\mathbf{2 0 0 8}$ & 0.0472 & 0.0908 & 0.1022 & 0.0839 & -0.0199 & 0.1170 & 0.0881 & 0.1581 & -0.3918 \\
\hline $\mathbf{2 0 0 9}$ & 0.1958 & -0.0891 & 0.0449 & -0.1693 & 0.0475 & -0.0684 & -0.2308 & -0.0438 & 0.8324 \\
\hline $\mathbf{2 0 1 0}$ & 0.1166 & -0.0003 & 0.3269 & 0.1560 & -0.0330 & -0.0429 & 0.1334 & -0.0050 & 0.5185 \\
\hline $\mathbf{2 0 1 1}$ & 0.1328 & 0.0997 & -0.2544 & 0.1160 & 0.0038 & 0.1060 & 0.1852 & 0.1329 & 0.1328 \\
\hline $\mathbf{2 0 1 2}$ & 0.0636 & -0.0569 & -0.0694 & -0.0664 & -0.0142 & -0.0856 & -0.0455 & -0.0324 & -0.3284 \\
\hline $\mathbf{2 0 1 3}$ & 0.0465 & 0.0588 & -0.0574 & 0.0560 & 0.0338 & 0.0752 & 0.0303 & 0.0415 & 0.0297 \\
\hline $\mathbf{2 0 1 4}$ & 0.0578 & 0.0368 & & 0.0361 & 0.0075 & 0.0016 & 0.0358 & 0.0589 & -0.7519 \\
\hline Average & 0.0276 & 0.0362 & 0.1739 & 0.0461 & 0.0304 & 0.0511 & 0.0637 & 0.0436 & 0.7431 \\
\hline STD & 0.1038 & 0.0907 & 0.8663 & 0.1064 & 0.1105 & 0.1041 & 0.1168 & 0.0879 & 3.9124 \\
\hline
\end{tabular}

Table 4. Summary of Japan Data

\begin{tabular}{|c|c|c|c|c|c|c|c|c|c|}
\hline Year & $\mathbf{G D P}_{\mathbf{M}}$ & GDP & INVEST $_{M}$ & PRODUCT $_{M}$ & $\mathbf{G D P}_{\mathbf{F}}$ & $\mathbf{G D P}_{\mathbf{R}}$ & EXPORT $_{M}$ & R\&D & FDI \\
\hline 1995 & 0.1097 & 0.1105 & 0.1232 & 0.1403 & 0.0524 & 0.1105 & 0.1120 & 0.1505 & -0.9569 \\
\hline 1996 & -0.1141 & -0.1129 & -0.0838 & -0.1079 & -0.0769 & -0.1172 & -0.0758 & -0.0845 & 4.2792 \\
\hline 1997 & -0.0874 & -0.0867 & -0.1137 & -0.0855 & -0.0944 & -0.0829 & 0.0211 & -0.0603 & 14.4115 \\
\hline 1998 & -0.1069 & -0.0866 & -0.1304 & -0.0674 & -0.1247 & -0.0720 & -0.0815 & -0.0523 & 0.0213 \\
\hline 1999 & 0.1101 & 0.1313 & 0.0759 & 0.1408 & 0.1241 & 0.1592 & 0.0756 & 0.1389 & 2.7662 \\
\hline 2000 & 0.0755 & 0.0713 & 0.0788 & 0.0942 & 0.0679 & 0.0736 & 0.1441 & 0.0761 & -0.1316 \\
\hline 2001 & -0.1704 & -0.1195 & -0.1436 & -0.1458 & -0.0305 & -0.1209 & -0.1673 & -0.0994 & -0.5391 \\
\hline 2002 & -0.0604 & -0.0438 & -0.1124 & 0.0007 & 0.0418 & -0.0390 & 0.0348 & -0.0302 & 1.3462 \\
\hline 2003 & 0.0920 & 0.0803 & 0.0690 & 0.1139 & 0.1236 & 0.0817 & 0.1331 & 0.0907 & -0.2410 \\
\hline 2004 & 0.0945 & 0.0831 & 0.0810 & 0.1214 & 0.0602 & 0.0820 & 0.1946 & 0.0783 & -0.1418 \\
\hline 2005 & 0.0021 & -0.0124 & 0.0039 & 0.0087 & -0.0112 & -0.0032 & 0.0427 & 0.0370 & -0.2748 \\
\hline 2006 & -0.0487 & -0.0473 & -0.0474 & -0.0636 & -0.0783 & -0.0239 & 0.0759 & -0.0181 & -1.4390 \\
\hline 2007 & 0.0180 & -0.0033 & -0.0140 & 0.0138 & -0.0116 & 0.0033 & 0.0932 & 0.0153 & $10.0246^{-}$ \\
\hline 2008 & 0.0830 & 0.1158 & 0.1187 & 0.1034 & -0.0496 & 0.1595 & 0.0835 & 0.1149 & 0.1384 \\
\hline 2009 & -0.0722 & 0.0384 & -0.0980 & -0.0106 & 0.0572 & 0.1275 & -0.2668 & 0.0055 & -0.5035 \\
\hline 2010 & 0.1856 & 0.0896 & 0.0883 & 0.2128 & 0.0605 & 0.0699 & 0.3401 & 0.0578 & -0.3914 \\
\hline 2011 & 0.0197 & 0.0802 & 0.1211 & 0.0492 & 0.0541 & 0.0999 & 0.0705 & 0.1173 & -1.1143 \\
\hline 2012 & 0.0108 & 0.0074 & 0.0325 & -0.0016 & -0.0287 & -0.0024 & -0.0248 & -0.0036 & -1.6429 \\
\hline 2013 & -0.1813 & -0.1689 & -0.1492 & -0.1870 & -0.1601 & -0.1753 & -0.1182 & -0.1414 & 18.4683 \\
\hline 2014 & -0.0467 & -0.0595 & -0.0315 & -0.0479 & -0.0901 & -0.0766 & -0.0343 & -0.0350 & 0.8549 \\
\hline 2015 & -0.0657 & -0.0960 & -0.0951 & -0.0613 & -0.1118 & -0.1196 & -0.0969 & -0.1266 & -0.7172 \\
\hline 2016 & 0.0052 & 0.1271 & 0.1004 & & & & 0.0382 & & 5.2492 \\
\hline Average & -0.0073 & -0.0014 & -0.0108 & 0.0105 & -0.0108 & 0.0064 & 0.0264 & 0.0110 & 1.1508 \\
\hline STD & 0.1000 & 0.0908 & 0.0970 & 0.1054 & 0.0831 & 0.1014 & 0.1346 & 0.0876 & 5.7371 \\
\hline
\end{tabular}

\subsection{Correlations}


The following tables provide correlations among selected variables of these four countries. It is clear that in all four countries, the $\mathrm{GDP}_{\mathrm{M}}$ and GDP are very highly correlated. This is obvious since a country's manufacturing and its total economy are usually co-integrated. In China, EXPORT $_{M}$ has the second highest correlation; in USA, INVEST ${ }_{M}$ and EXPORT $_{M}$ have high correlations with the $\mathrm{GDP}_{M}$. In Germany, EXPORT $\mathrm{E}_{\mathrm{M}}$ and $\mathrm{PRODUCT}_{\mathrm{M}}$ have high correlations; and in Japan, INVEST ${ }_{M}$ and PRODUCT ${ }_{M}$ have high correlations with its GDP $_{M}$.

Table 5. China Variables Correlations

\begin{tabular}{|r|r|r|r|r|r|r|r|r|r|}
\hline & GDP $_{\mathbf{M}}$ & GDP & INVEST $_{\mathbf{M}}$ & PRODUCT $_{\mathbf{M}}$ & GDP $_{\mathbf{F}}$ & GDP $_{\mathbf{R}}$ & EXPORT $_{\mathbf{M}}$ & R\&D & FDI \\
\hline GDP $_{\mathbf{M}}$ & 1.000 & 0.967 & 0.445 & 0.309 & 0.212 & 0.564 & 0.602 & 0.484 & 0.122 \\
\hline GDP & 0.967 & 1.000 & 0.358 & 0.339 & 0.268 & 0.490 & 0.511 & 0.497 & -0.003 \\
\hline INVEST $_{\mathbf{M}}$ & 0.445 & 0.358 & 1.000 & -0.501 & -0.591 & 0.173 & 0.653 & 0.240 & 0.142 \\
\hline PRODUCT $_{\mathbf{M}}$ & 0.309 & 0.339 & -0.501 & 1.000 & 0.825 & 0.176 & -0.288 & 0.243 & -0.261 \\
\hline GDP $_{\mathbf{F}}$ & 0.212 & 0.268 & -0.591 & 0.825 & 1.000 & 0.209 & -0.459 & 0.274 & -0.237 \\
\hline GDP $_{\mathbf{R}}$ & 0.564 & 0.490 & 0.173 & 0.176 & 0.209 & 1.000 & 0.252 & -0.159 & 0.619 \\
\hline EXPORT $_{\mathbf{M}}$ & 0.602 & 0.511 & 0.653 & -0.288 & -0.459 & 0.252 & 1.000 & 0.362 & 0.309 \\
\hline R\&D & 0.484 & 0.497 & 0.240 & 0.243 & 0.274 & -0.159 & 0.362 & 1.000 & -0.310 \\
\hline FDI & 0.122 & -.003 & 0.142 & -0.261 & -0.237 & 0.619 & 0.309 & -0.310 & 1.000 \\
\hline
\end{tabular}

Table 6. USA Variables Correlations

\begin{tabular}{|r|r|r|r|r|r|r|r|r|r|}
\hline & GDP $_{\mathbf{M}}$ & GDP & INVEST $_{\mathbf{M}}$ & PRODUCT $_{\mathbf{M}}$ & GDP $_{\mathbf{F}}$ & GDP $_{\mathbf{R}}$ & EXPORT $_{\mathbf{M}}$ & R\&D $_{\mid}$ & FDI \\
\hline GDP $_{\mathbf{M}}$ & 1.000 & 0.783 & 0.661 & 0.315 & 0.208 & 0.097 & 0.789 & 0.359 & 0.643 \\
\hline GDP & 0.783 & 1.000 & 0.534 & 0.110 & 0.332 & 0.550 & 0.689 & 0.611 & 0.502 \\
\hline INVEST $_{\mathbf{M}}$ & 0.661 & 0.534 & 1.000 & -0.336 & -0.079 & -0.094 & 0.760 & 0.549 & 0.474 \\
\hline PRODUCT $_{\mathbf{M}}$ & 0.315 & 0.110 & -0.336 & 1.000 & 0.043 & -0.007 & 0.167 & -0.237 & 0.159 \\
\hline GDP $_{\mathbf{F}}$ & 0.208 & 0.332 & -0.079 & 0.043 & 1.000 & 0.188 & -0.124 & -0.017 & -0.070 \\
\hline GDP $_{\mathbf{R}}$ & 0.097 & 0.550 & -0.094 & -0.007 & 0.188 & 1.000 & 0.134 & 0.403 & -0.105 \\
\hline EXPORT $_{\mathbf{M}}$ & 0.789 & 0.689 & 0.760 & 0.167 & -0.124 & 0.134 & 1.000 & 0.445 & 0.467 \\
\hline R\&D & 0.359 & 0.611 & 0.549 & -0.237 & -0.017 & 0.403 & 0.445 & 1.000 & 0.341 \\
\hline FDI & 0.643 & 0.502 & 0.474 & 0.159 & -0.070 & -0.105 & 0.467 & 0.341 & 1.000 \\
\hline
\end{tabular}

Table 7. Germany Variables Correlations

\begin{tabular}{|r|r|r|r|r|r|r|r|r|r|}
\hline & GDP $_{\mathbf{M}}$ & GDP & INVEST $_{\mathbf{M}}$ & PRODUCT $_{\mathbf{M}}$ & GDP $_{\mathbf{F}}$ & GDP $_{\mathbf{R}}$ & EXPORT $_{\mathbf{M}}$ & R\&D $_{\mid}$ & FDI \\
\hline $\mathbf{G D P}_{\mathbf{M}}$ & 1.000 & 0.900 & 0.130 & 0.964 & 0.475 & 0.755 & 0.924 & 0.861 & -0.038 \\
\hline GDP & 0.900 & 1.000 & 0.158 & 0.917 & 0.663 & 0.947 & 0.772 & 0.947 & -0.041 \\
\hline INVEST $_{\mathbf{M}}$ & 0.130 & 0.158 & 1.000 & 0.151 & 0.157 & 0.165 & 0.223 & 0.132 & -0.101 \\
\hline PRODUCT $_{\mathbf{M}}$ & 0.964 & 0.917 & 0.151 & 1.000 & 0.533 & 0.820 & 0.882 & 0.848 & 0.026 \\
\hline GDP $_{\mathbf{F}}$ & 0.475 & 0.663 & 0.157 & 0.533 & 1.000 & 0.592 & 0.301 & 0.635 & -0.161 \\
\hline GDP $_{\mathbf{R}}$ & 0.755 & 0.947 & 0.165 & 0.820 & 0.592 & 1.000 & 0.629 & 0.844 & 0.014 \\
\hline EXPORT $_{\mathbf{M}}$ & 0.924 & 0.772 & 0.223 & 0.882 & 0.301 & 0.629 & 1.000 & 0.740 & 0.093 \\
\hline R\&D & 0.861 & 0.947 & 0.132 & 0.848 & 0.635 & 0.844 & 0.740 & 1.000 & -0.108 \\
\hline FDI & -0.038 & -0.041 & -0.101 & 0.026 & -0.161 & 0.014 & 0.093 & -0.108 & 1.000 \\
\hline
\end{tabular}


Table 8. Japan Variables Correlations

\begin{tabular}{|r|r|r|r|r|r|r|r|r|r|}
\hline & GDP $_{\mathbf{M}}$ & GDP & INVEST $_{\mathbf{M}}$ & PRODUCT $_{\mathbf{M}}$ & GDP $_{\mathbf{F}}$ & GDP $_{\mathbf{R}}$ & EXPORT $_{\mathbf{M}}$ & R\&D & FDI \\
\hline GDP $_{\mathbf{M}}$ & 1.000 & 0.918 & 0.916 & 0.980 & 0.728 & 0.819 & 0.840 & 0.875 & -0.429 \\
\hline GDP & 0.918 & 1.000 & 0.909 & 0.944 & 0.822 & 0.973 & 0.633 & 0.965 & -0.443 \\
\hline INVEST $_{\mathbf{M}}$ & 0.916 & 0.909 & 1.000 & 0.880 & 0.657 & 0.820 & 0.741 & 0.918 & -0.397 \\
\hline PRODUCT $_{\mathbf{M}}$ & 0.980 & 0.944 & 0.880 & 1.000 & 0.799 & 0.866 & 0.786 & 0.893 & -0.437 \\
\hline GDP $_{\mathbf{F}}$ & 0.728 & 0.822 & 0.657 & 0.799 & 1.000 & 0.797 & 0.496 & 0.787 & -0.410 \\
\hline GDP $_{\mathbf{R}}$ & 0.819 & 0.973 & 0.820 & 0.866 & 0.797 & 1.000 & 0.490 & 0.937 & -0.425 \\
\hline EXPORT $_{\mathbf{M}}$ & 0.840 & 0.633 & 0.741 & 0.786 & 0.496 & 0.490 & 1.000 & 0.647 & -0.241 \\
\hline R\&D & 0.875 & 0.965 & 0.918 & 0.893 & 0.787 & 0.937 & 0.647 & 1.000 & -0.409 \\
\hline FDI & -0.429 & -0.443 & -0.397 & -0.437 & -0.410 & -0.425 & -0.241 & -0.409 & 1.000 \\
\hline
\end{tabular}

III. The Econometric model, hypotheses, and regression outcomes

\subsection{Econometric model:}

$\mathrm{GDP}_{\mathrm{M}}=\mathrm{a}+\mathrm{b} * \operatorname{INVEST}_{\mathrm{M}}+\mathrm{c} * \operatorname{PRODUCT}_{\mathrm{M}}+\mathrm{d} * \mathrm{GDP}_{\mathrm{F}}+\mathrm{e}^{*} \mathrm{GDP}_{\mathrm{R}}++\mathrm{f}^{*}$ EXPORT $_{\mathrm{M}}+\mathrm{g} * \mathrm{R} \& D+\mathrm{h} * \mathrm{FDI}$

where all variables are as explained before; $\mathrm{a}, \mathrm{b}, \mathrm{c}, \mathrm{d}, \mathrm{e}, \mathrm{f}, \mathrm{g}$, and $\mathrm{h}$ are coefficients to be estimated.

Besides INVEST $\mathrm{M}, \mathrm{PRODUCT}_{\mathrm{M}}, \mathrm{GDP}_{\mathrm{F}}, \mathrm{GDP}_{\mathrm{R}}$, and EXPORT $\mathrm{M}$, two control variables-R\& D and FDI are included. An economy's R \& D spending may directly affect its growth; since the R\& D spending in manufacturing is not available, the total $R \& D$ spending in the country is used. The annual growth rate of total R\&D spending in a country should be considered as the proxy for the annual growth of R \& D in manufacturing. Also, FDI may directly influence an economy's development. Similarly, the annual growth rates of the total FDI are used, instead of the annual growth rate of FDI in manufacturing.

\subsection{Hypotheses}

Hypothesis one-the effect of the financial industry on the manufacturing industry. The null hypothesis is no significant relation between the $\mathrm{GDP}_{\mathrm{F}}$ and $\mathrm{GDP}_{\mathrm{M}}$ or

$\mathrm{d}=0$; and the alternative hypothesis is

$\mathrm{d} \neq 0$;

Hypothesis two-the effect of the real estate industry on the manufacturing industry. The null hypothesis is no significant relation between the $\mathrm{GDP}_{\mathrm{R}}$ and $\mathrm{GDP}_{\mathrm{M}}$ or

$\mathrm{e}=0$; and the alternative hypothesis is

$\mathrm{e} \neq 0$;

Hypothesis three-the effect of manufacturing productivity on its growth. The null hypothesis is no significant relation between the PRODUCT $\mathrm{M}$ and $\mathrm{GDP}_{\mathrm{M}}$ or

$\mathrm{c}=0$; and the alternative hypothesis is

$\mathrm{c} \neq 0$;

Hypothesis four- the effect of manufacturing export on its growth. The null hypothesis is no significant relation between the EXPORT $\mathrm{M}_{\mathrm{M}}$ and $\mathrm{GDP}_{\mathrm{M}}$ or

$\mathrm{f}=0$; and the alternative hypothesis is

$\mathrm{f} \neq 0$.

Besides Hypotheses one and two, Hypothesis three is added because the productivity of the sector may affect its growth and attractiveness to the investors as previous studies indicated. Hypothesis four also is added because for export-oriented economy like China or Germany, its export change should be crucial to its manufacturing growth.

\subsection{Regression outcomes}

The following table is the summary of model regressions for China, USA, Germany and Japan. 
Table 9. Summaries of Model Regressions

\begin{tabular}{|c|c|c|c|c|}
\hline & China & USA & Germany & Japan \\
\hline Constant (a) & -0.0888 & -0.0216 & $* *$-0.0205 & $*_{-0.0124}$ \\
\hline INVEST $_{M}(\mathbf{b})$ & 0.0447 & $* 0.2208$ & -0.0059 & $* 0.3053$ \\
\hline PRODUCT $_{M}(c)$ & 0.4346 & $* 0.5501$ & $* * 0.5854$ & $* * 0.7292$ \\
\hline $\operatorname{GDP}_{\mathrm{F}}(\mathbf{d})$ & 0.4346 & 0.1399 & -0.0179 & -0.0003 \\
\hline $\operatorname{GDP}_{\mathbf{R}}(\mathbf{e})$ & 0.2948 & 3163 & -0.0879 & 0.0530 \\
\hline EXPORT $_{M}(\mathbf{f})$ & $* 0.3169$ & 0.0373 & $* 0.2927$ & 0.0974 \\
\hline R \& D (g) & 0.0571 & -0.1682 & 0.2285 & -0.2156 \\
\hline FDI (h) & -0.033 & 0.0172 & -0.0018 & -0.0003 \\
\hline Number of Samples & 23 & 18 & 22 & 21 \\
\hline F-statistic & $* * 11.3144$ & $* * 11.7759$ & $* * 65.6856$ & $* * 136.7099$ \\
\hline
\end{tabular}

$* 5 \%$ significant and $* * 1 \%$ significant

The above table shows that for Hypotheses one and two, both nulls are accepted; in other words, there are no significant relationships between the financial or real estate industry and the manufacturing industry in all these four countries or there is no evidence that the fast development of the financial industry or real estate sector will negatively affect the manufacturing growth.

For Hypothesis three, except China, alternative/research hypothesis is accepted. In other words, the manufacturing productivity growth significantly increased its manufacturing GDP growth in USA, Germany and Japan.

For Hypothesis four, the alternative/research hypothesis is accepted for both China and Germany, which means that the manufacturing export growth significantly raised its GDP growth in manufacturing in China and Germany. In addition, the above regression outcome indicates that the manufacturing investment growth significantly raised its manufacturing GDP growth in USA and Japan.

\section{Policy implications}

Based on the data used and regression results, the developments of the financial and real estate sectors are not to be blamed for the slowing down of the manufacturing in all these countries. Instead, the productivity of the manufacturing industry is more important and significant to the change of the manufacturing industry. Therefore, one should focus more on improving the productivity of the industry by adopting advanced technologies, innovations, better employee training and decreasing of the operation and transaction costs.

Investment in the manufacturing industry is another factor, particularly in the USA and Japan. In order to maintain a stable growth of the industry, a country needs to continuously attract more investments. Then, less regulation or some deregulation by the governments will help the industry.

Also, for export-oriented countries like China and Germany, its global competitiveness of products and therefore increasing of exports will be essential to its manufacturing stability and growth. Thus, uniqueness, the intensity level of capital and technology, and brands/reputations of the products are important because loyalty of the consumers will be critical to the steady growth of the manufacturing industry.

Given its challenges and difficulties of manufacturing export, China should learn from USA and Japan and focus more on improving its productivity of the manufacturing sector and attracting more investments to the sector in order to stabilize and strengthen its manufacturing industry. Then, China should further open its door and deepen its reforms to better encourage entrepreneurship and innovations and attract more foreign investments in the manufacturing industry.

The paths toward a stable manufacturing industry and sustainable developments are different. Countries rely on its different and unique advantages to uphold its competitiveness. In addition, in its different development stage, a country needs to take a different path as explained above in the case of China.

\section{Conclusions and future research}

This paper studies the relationship of the manufacturing industry with the financial and real estate industry. Based on the data from China, USA, Germany, and Japan, it used the multiple regression model to identify factors/variables significantly affecting the manufacturing growth and concluded that there are no such direct relationships between the 
manufacturing and financial or real estate sectors. On the other hand, the regression results indicated that the productivity and export of the manufacturing industry and investment in that industry have more significant effects on the manufacturing's sustainable growth. Then relevant policy implementations are further discussed.

The data used in this paper are limited. One may run a fix effect or random effect model if the needed panel data are available. In addition, one may use two-stage simultaneous regression models to analyze the effects of the relevant variables on the manufacturing growth.

\section{Acknowledgements and Endnotes}

Authors want to thank financial support from their universities, particularly Henan Province Higher Education Philosophy and Social Sciences Research Fund (\# 2019-YYZD-05).

* ( the first two) are corresponding authors.

The data from the World Bank is at www.worldbank.org. China's investment data in manufacturing are from its National Bureau of Statistics at www.stats.gov.cn/english; USA from its Census Office at www.census.gov/programssurveys/aces.html; Germany from sdw.ecb.europa.eu; and Japan from www.theglobaleconomy.com/ Japan/capital_investment_dollars .

\section{References}

Anwar, S. \& Sun, S. (2013), "Foreign Entry and Firm R\&D: Evidence from Chinese Manufacturing Industries," $\boldsymbol{R} \& \boldsymbol{D}$ Management, Volume 43(4), 303-317;

Arnold, J. M., Javorcik, B., Lipscomb, M., \&Mattoo, A. (2014), "Services Reform and Manufacturing Performance: Evidence from India," The Economic Journal, Volume 126(590), 1-39;

Atje, R. \&Jovanovic, B. (1993), "Stock Markets and Development," European Economic Review, Volume 37(2-3), 632-640;

Baily, M. N. \& Bosworth, B. P. (2014), "US Manufacturing: Understanding Its Past and Its Potential Future,” Journal of Economic Perspectives, Volume 28(1), 3-26;

Baldwin, J. R., Gu, W., \& Yan, B. (2013), "Export Growth, Capacity Utilization, and Productivity Growth: Evidence from the Canadian Manufacturing Plants," The Review of Income and Wealth, Volume 59(4), 665-688;

Bencivenga, V. R. \& Smith, B. D. (1991), "Financial Intermediation and Endogenous Growth, The Review of Economic Studies, Volume 58(2), 195-209;

Chen, Bill Y., et al. (2017), "The Productivity, Economic Structure and Middle-Income Trap - Can China Avoid this Trap?" Journal of Applied Business and Economics, Volume 19(11), 79-104;

Chen, Bill Y. (2015), "China's Path to the Sustainable, Stable and Rapid Economic Development: From the Largest to the Strongest Manufacturing Country"; Journal of World Economic Research; Volume 4 ( 5-1), 8-19;

Cezar, Rafael (2014), "The Heterogeneous Effect of Finance on International Trade," Applied Economics; Volume 46(24), 2903-2919;

De, Prasanta Kumar (2014), "Technological Competitiveness of the Danish Manufacturing Industry: A Critical Review," South Asian Journal of Management, Volume 21(4), 100-119;

Dehejia, R. H. \&Panagariya, A. (2014), “The Link Between Manufacturing Growth and Accelerated Services Growth in India," Economic Development and Cultural Change, Volume 64(2), 221-264;

Goldsmith, R.W. (1969), Financial Structure and Development; Yale University Press, New Haven;

Greenwood, J. \& Smith, B. D. (1997), "Financial Markets in Development, and the Development of Financial Markets," Journal of Economic Dynamics and Control, Volume 21(1), 145-181;

Jung, Woo S. (1986), "Financial Development and Economic Growth: International Evidence," Economic Development and Cultural Change, Volume 34(2), 333-346;

Kerwin, K. C., Hurst, E.\&Notowidigdo, M. J. (2016), "The Masking of the Decline in Manufacturing Employment by the Housing Bubble," The Journal of Economic Perspectives, Volume 30(2), 179-200;

King, R. G., and Levine, R.(1992), "Financial Indicators and Growth in a Cross Section of Countries," World Bank Working Paper No. 819;

King, R. G. \& Levine, R. (1993a), "Finance and Growth: Schumpeter Might Be Right," The Quarterly Journal of Economics, Volume 108(3), 717-737;

King, R. G. \& Levine, R. (1993b), "Finance, Entrepreneurship, and Growth: Theory and Evidence," Journal of Monetary Economics, Volume 32(3), 513-542;

Kim, Sangho (2015), "Factor Determinants of Total Factor Productivity Growth for the Japanese Manufacturing Industry," Contemporary Economic Policy, Volume 34(3), 572-586; 
Lucus. R.E. (1988), “ On the Mechanics of Economic Development,”Journal of Monetary Economics, 22 (1988) 342 ;

Neusser, Klaus and Maurice Kugler (1998), "Manufacturing growth and financial development: evidence from OECD countries," Review of Economics and Statistics, Volume 80 (4), 638-646;

Pagano, Marco (1993), "Financial Markets and Growth: An Overview," European Economic Review, Volume 37(2-3), 613-622;

McKinnon, R.I. (1973), Money and Capital in Economic Development, Washington, DC:

Brookings Institution;

Pai, Mi Kyung (2016), "The Technical Progress and Resilience in Productivity Growth of Korea's Growth-Leading Industries," Asian Economic Papers, Volume 15(2), 167-191;

Paul, Justin (2016), “The Rise of China: What, When, Where, and Why?" The International Trade Journal, Volume 30(3), 207-222;

Robinson. J. (1952) "The Generalization of the General Theory," The Rate of interest and other essays. London: Macmillan;

Roubini, N. \& Sala-i-Martin, X. (1992), "Financial Repression and Economic Growth," Journal of Development Economics, Volume 39(1), 5-30;

Shaw, E.S. (1973), Financial Deepening in Economic Development, New York: Oxford

University Press;

Solow, Robert M. (1957), "Technical Change and the Aggregate Production Function," Review of Economics and Statistics,39 (3): 312-320;

Solow. R.M. (1956), A Contribution to the Theory of Economic Growth; The Quarterly Journal of Economics, Vol. 70, No. 1. 65-94;

Stern. N. (1989), “The Economics of Development: A Survey,” The Economic Journal, Vol.99, No.397, 597-685;

Vukšić, Goran (2015), "Effects of Private Ownership, Trade, and Foreign Direct Investment on Labor Productivity Growth in Transition Economies: Evidence from the Croatian Manufacturing Industry," Emerging Markets Finance\& Trade, Volume 52(2), 322-335. 